\title{
Design and fabrication of a dc driver circuit control dc motor
}

\author{
Diponkar Paul*, Rajib Mondal \\ Dept of EEE World University of Bangladesh
}

Email address:

dipo0001@ntu.edu.sg (D. Paul)

\section{To cite this article:}

Diponkar Paul, Rajib Mondal. Design and Fabrication of a De Driver Circuit Control Dc Motor. International Journal of Energy and Power Engineering. Vol. 2, No. 1, 2013, pp. 1-11. doi: 10.11648/j.ijepe.20130201.11

\begin{abstract}
A DC Motor Driver is a device that drives motor, typically as a speed controller of motor. This DC Motor Driver circuit uses a 741 operational amplifier operating as a voltage follower where its non inverting input is connected to the speed and rotation direction of a potentiometer. When the potentiometer is turned towards the positive supply side, the output will go positive voltage and NPN transistor will supply the current to the motor and PNP transistor will be OFF. When the potentiometer is turned to the negative supply side, the op-amp output switches to the negative voltage and NPN Transistor will turn OFF and PNP Transistor ON which reverses the rotation of the motor's direction. It has been design along with Op-Amp to drive the motor. The fabrication results of DC Motor Driver provide very good results. The total cost of fabricated our dc motor driver using Op-Amp is comparatively cheaper than the traditional dc Motor Driver available in market price. A DC Motor specification is taken and corresponding parameters are found out from derived design approach. Ultimately simulation is done for model with and without filter used after reference speed and a comparative study has been done on response of both cases. Further it is purposed that in industrial environment these controllers are better than others controllers as Op-Amp controllers are easy and cheap.
\end{abstract}

Keyword: Dc Driver Circuit, TB+, Half Cycle

\section{Introduction}

This report presents a new way of control engineering. Dc Motor Speed controlled by 741 Op-Amp, pole placement and $741 \mathrm{Op}$-Amp and discusses the advantages of each controller for different conditions under loaded and unloaded. The brushless series wound Dc motor is very popular in industrial application and control systems because of the high torque density, high efficiency and small size. First suitable equations are developed for DC motor. 741 Op-Amp controllers is developed and tuned in order to get faster step response. The fabrication results of dc motor driver provide very good results and the controller is further tuned in order to decrease its overshoot error. Further it is purposed that in industrial environment these controllers are better than others controllers as OpAmp controllers are easy and cheap. Pole placement controller is the best example of control engineering. An addition of integrator reduced the noise disturbances in pole placement controller and this makes it a good choice for industrial applications. The method of speed control system that will be used in our Engineering sections,
Industrial sectors etc. DC Driver Circuit has a long tradition of use as adjustable speed machines and a wide range of options have evolved for this purpose. In the enormous penetration of power supplies and their component parts at every level of electronic products and systems. So, DC Motor driver is needed where it can control the magnitude of supply voltage in order to control the speed of DC motor. As the potentiometer is moved toward either end, the speed increases in whichever direction it is turning. If a DC Motor is connected directly to the voltage supply, the constant power will be supplied to the DC motor all the time. DC drives are normally less expensive for most horsepower ratings. Permanent-magnet dc motors are limited to rating of a few horsepower and also have a maximum speed limitation. These can be overcome if Qf is produced by means of a field winding on the stator, which is supplied by a dc current If . To offer the most flexibility in controlling the dc motor, the field winding is excited by a separately controlled dc source Vf. As indicated by $\mathrm{Eq}$, the steady-state value of $\mathrm{Qf}$ is controlled by If(=VflRf), s kept at its rated value for speeds less than the rated speed.With Qf at its rated value, 
Therefore, the torque-speed characteristics are also the same as those for a permanent-magnet dc-motor. With Qf constant and equal to its rated value, the motor- torquespeed capability where this region of constant Qf is often called the constant-torque region. The required terminal voltage $\mathrm{Vt}$ in this region increase linearly from approximately zero to its rated value as the speed increases from zero to its rated value. The voltage $\mathrm{Vt}$ and the corresponding Ea. To obtain speeds beyond its rated value, $\mathrm{Vt}$ is kept constant at its rated value and Qf is decreased by decreasing If. Since Ia is not allowed to exceed its rated value on a continuous basic, the torque capability decline, since Qf is reduced in Eq. In this so-called field-weakening region, the maximum power Ea Ia into the motor is not allowed to exceed its rated value on a continuous basis. This region, also called the constant-power region. Where Tem declines with $\mathrm{Wm}$ and $\mathrm{Vt}$, Ea and Ia stay constant at their rated values. It should be emphasized is the plot of the maximum continuous capability of the motor in steady state. Any operating point within shown is, of course, permissible. In the field-weakening region, the speed may be exceeded by $50-100 \%$ of its rated value, depending on the motor specifications. In dc motor drives, the output voltage of the power electronic converter contains an ac ripple voltage superimposed on the desired dc voltage. Ripple in the terminal voltage can lead to a ripple in the armature current with the following consequences that must be recognized: the from factor and torque pulsations. The form factor will be unity only if Ia is a pure dc. The more Ia deviates from a pure dc, the higher will be the value of the form factor. The power input to the motor varies proportionally with the average value of Ia, whereas the losses in the resistance of the armature winding depend on I2a (rms). Therefore, the higher the form factor of the armature current, the higher the losses in the motor and hence, the lower the motor efficiency. Moreover, a form factor much higher than unity implies a much larger value of the peak armature current compared to its average value, which may result in excessive arcing in the commentator and brushes. To avoid serious damage to the motor that is caused by large peak currents, the motor may have to be dated (i.e, the maximum power or torque would have to be kept well below its rating) to keep the motor temperature from exceeding its specified limits and to protect the commentator and brushes. Therefore, it is desirable to improve that form factor of the armature current as much as possible. In servo application, the speed and accuracy of response is important. In spite of the increasing popularity of ac servo drives, dc servo drives are still widely used. If it were not for the disadvantages of having a commentator and brushes, the dc motors would be ideally suited for servo drives. The reason is that the instantaneous torque Tem in Eq.13-2 can be controlled linearly by controlling the armature current ia of the motor. To design the proper controller that will result in high performance (high speed of response, low steady-state error, and high degree of stability), it is important to know the transfer function of the motor. It is then combined with the transfer function of the system in order to determine the dynamic response of the drive for change in the desired speed and position or for a change in load. As we will explain later on, the linear model is valid only for small changes where the motor current is not limited by the converter supplying the motor. For analyzing small signal dynamic performance of the motor load combination around a steady state operating point, the following equation can be written in terms of small deviations around their steady state value.

\section{Case Studies}

DC motor drives are defined as amplifiers or power modules that interface between a controller and a dc motor. They convert step and direction input from the controller to currents and voltages compatible with the motor. These units are sometimes called variable speed drives. $741 \mathrm{Op}$ Amp based on DC Motor Controller Circuit. This DC Motor Driver Circuit uses 741 operational amplifiers operating as a voltage follower where its non inverting input is connected to the speed and rotation direction of a potentiometer VR1. This $9 \mathrm{~V}$ dc is given as the voltage source of op-amp. Then connect VCC supply Pin 7, and GND pin 4 as the negative input of Op-Amp. As the potentiometer VR1 is moved toward either end, the speed increases in whichever direction it is turning. If a DC motor is connected directly to the voltage supply, the constant power will be supplied to the DC motor all the time. When the potentiometer is turned towards the positive supply side, the output will go positive voltage and NPN transistor will supply the current to the motor and PNP transistor will be OFF. When the potentiometer is turned to the negative supply side, the op-amp output switches to the negative voltage and NPN Transistor will turn OFF and PNP Transistor ON which reverses the rotation of the motor's direction. DC motor in inherently bidirectional. Hence no problem to reverse the direction. It can be a motor or generator. From the derivation, several important facts can be deduced for steady-state operation of DC motor. For a fixed field current, or flux (If), the torque demand can be satisfied by varying the armature current (Ia). These observations lead to the application of variable DC voltage to control the speed and torque of DC motor. These observations lead to the application of variable DC voltage to control the speed and torque of DC motor. For Example, Consider a $500 \mathrm{~V}, 10 \mathrm{~kW}, 20 \mathrm{~A}$ ratedDC motor with armature resistance of $1 \mathrm{ohm}$. When supplied at $500 \mathrm{~V}$, the UNLOADED motor runs at 1040 $\mathrm{rev} / \mathrm{min}$, drawing a current of $0.8 \mathrm{~A}$ (ideally current is zero at no-load). In order to operate power semiconductor devices at high switching frequencies, drive circuits must be designed to turn-off the devices as rapidly as they turn on. The description of the switching characteristics of BJTs, MOSFET, IGBTs, and other device clearly illustrated the 
need for a reverse biased to be applied to the control terminal of the power switch in order to affect a rapid turn off. Drive circuits with unipolar outputs are unable to provide the needed reverse bias and thus are incapable of providing first turn off of power devices. In order to provide a reverse bias to the control terminal of the power device, the driver circuits must have a bipolar output .This in turn requires that the drive circuit be biased by a negative power supply as well as positive power supply.

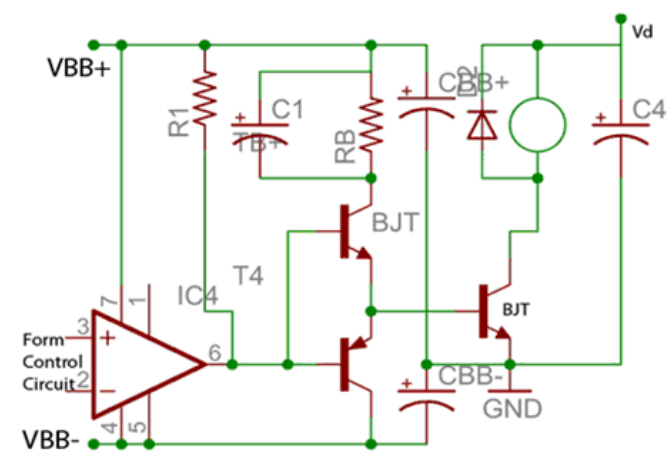

Fig. 2.1. (a) DC-Coupled Drive Circuit.

BJT drive circuit design applies in the selection of $\mathrm{VBB}+$ and RB. The optional capacitor Con shown as dashed acts as a speed-up capacitor by providing a large transient base current to the power transistor at the instant of turn-off to speed up the turn-on sequence. For turning the BJT off the internal output transistor of the comparator turns on thus turning the PNP transistor TB. For a fast turnoff no external resistance is used series with TB-.The magnitude of the negative voltage must be less than the $\mathrm{BE}$ breakdown voltage of the BJT, which is given on the data sheet and normally 5-7V range. The switching waveform will be similar to those described in section 21-5 if the BJT is used in a similar circuit. If the BJT has tendency for collector current tailing due to a too rapid turn-off of the $\mathrm{BE}$ junction compared to the $\mathrm{CB}$ junction, as described in section 21-5,then a resistor, if necessary, an inductor can be added in the turn-off base drive between points $\mathrm{A}$ and the emitter of TB-in Fig. a. If the control signal is supplied by a logic circuit that is connected between VBB+ and the emitter of the BJT, then the reference input to the compactor should be at the mid-pintail between $\mathrm{VBB}+$ and the BJT emitter terminal, as is shown in Fig.1.7(b) Where $\mathrm{R} 4=\mathrm{R} 5$. The modification shown in Fig.1.7c further enhance the BJT turn-off performance of the drive of Fig 2.17(d).An anti-saturation diode Das added to keep the BJT voltage Vce slightly above its saturation value Vce(sat). since $\mathrm{VBE}($ on) is in general large than $\mathrm{VCE}$ (sat), the presence of the anti-saturation diode keeps the transistor slightly out of saturation, thus reducing the storage time at the expanse of increased on-state losses in the BJT. Therefore the anti-saturation diode should only be used if the capability to use the BJT in a high switching frequency application is required. If still faster turn-off switching is needed, the on-state voltage VCE (sat) can be adjusted by putting one or more diodes in series withD1. IN the circuit of Fig.1.7(c), the diode D2 is needed to provide a path for the negative base current. Das should be a fast recovery diode with a reverse recovery time smaller than the storage time of the BJT. Moreover, its reverse voltage rating must be similar to the off-state voltage rating of the power transistor. An improved version of the circuit of Fig.1.7 (e) is shown in $28-5 \mathrm{~b}$ where the power loss in the positive portion of the base drive circuit is reduce compared to the original circuit. Here the anti -saturation diode adjusts the base current of the drive transistor $\mathrm{TB}+$ such that $\mathrm{TB}+$ operates in the active mode and the current drawn from $\mathrm{VBB}+$ now is only equal to the actual IB needed to barely saturate the BJT. Moreover the current rating required of Das is reduced. A small resistance in series with the antisaturation diode can significantly help reduce oscillations at turn-on. Since TB+ operates in the active region, it must be mounted on a small heat sink. A drive circuit for MOSFETs that provides positive gate voltage at turn on and negative gate voltage at turn-off by means of a spilt power supply with respect to the MOSFET source is shown in Figs.1.7(f) through 28-6c.If the control signal is supplied by logic circuit that is connected between VGG+ and the source of the MOSFET, then the reference input to the comparator should be shifted to be at the mid-potential between VGG+ and the source of the MOSFET using a pre-converter circuit similar to that in Fig.1.7b. Very often, there is a need for electrical isolation between the logic level control signals and the drive circuits. This is illustrated for the case of a power BJT half-bridge converter having a signal-phase ac supply as its input where one of the power terminals is a grounded neutral wire. Now the positive $\mathrm{dc}$ bus is close to the ground potential during the negative half cycle of Vs, and the negative dc bus is near ground potential during the positive half cycle of $\mathrm{Vs}$.Under these conditions the emitter terminals of both BJTs must be treated as hot with respect to power neutral. The logic-level control signals are the power neutral since the logic circuits are connected to the neutral of a safety ground wire. The basic ways to provide electrical isolation are either by optocouples fiber optics, or by transformers. The optocoupler consists of a lightemitting diode (LED) the output transistor and a built in Schmitt trigger. A positive signal from the control logic causes the LED to emit light that is focused on the optically sensitive base region of a photo transistor. The light falling on the base region generates a significant number of electron hole pairs in the base region that cause the photo transistor to turn on. The resulting drop in voltage at the photo transistor collector causes the Schmitt trigger to change state. The output of the Schmitt trigger is the optocoupler output and can be used as the control input to the isolated drive circuit. The capacitance between the LED. As an alternative, fiber optic cables can be used to completely eliminate this re-triggering problem and to 
provide very high electrical isolation and creep age distance. When using fiber optic cable the LED is kept on the circuit board of the control electronics and optical fiber transmits the signal to the receiver transistor, which is put on the drive circuit printed circuit board. Instead of using optocouplers or fiber optic cables, the control signal can be coupled to the electrically isolated drive circuit by means of a transformer as is shown in Fig 28-9a.If the switching frequency is high and the duty ratio D varies only slightly around 0.5 a base band control signal of appropriate pulse transformer as implied in Fig.1.7h, and the secondary output can be used to either directly drive the power switch or used as the input to an isolated drive circuit. As the switching frequency is below the tens of kilohertz rage a baseband control signal directly applied to the transformer primary becomes impractical because the size and weight of the transformer becomes increasing larger. Modulation of a high frequency carrier by a low frequency control signal enables a small high frequency pulse transformer to be used for even low frequency oscillator output before being applied to the primary of a high frequency signal transformer. Since a a high frequency transformer can be made quite small, it is easy to avoid stray capacitance between the input and the output winding, and the transformer will be inexpensive. The transformer secondary output is rectified and filtered and then applied to the comparator and the rest of the isolated drive circuit. The waveforms for this modulation scheme. Various gate drive circuits using split dc power supplies for providing an n-channel MOSFET with positive gate-source voltages at turn-on and negative gate drive at turn-off. Family of steady-state torque speed curves for a range of armature voltage can be drawn as above. The speed of DC motor can simply be set by applying the correct voltage. Note that speed variation from no-load to full load (rated) can be quite small. It depends on the armature resistance. Power is also negative, which implies power is "generated" back to the supply. In other words, during the deceleration phase, kinetic energy from the motor and load inertia is returned to the supply. This is known as regenerative braking-an efficient way to brake a motor. Widely employ in electric vehicle and electric trains. If we wish the motor to operate continuously at position $\mathrm{B}$, the machine have to be driven by mechanical source [1]. The dc motor control has played a vital role in the advance of engineering and science. Nowadays in industries, the control of direct current (DC) motor is a common practice thus the implementation of DC motor of controller speed is important. The main purpose of motor speed control is to keep the rotation of the motor at the preset speed and to drive a system at the demanded speed. When used in speed application, speed feedback control the DC motor's speed or confirms that the motor is rotating at the desired speed. To maintain the speed, it requires the speed feedback at all times. The speed of a DC motor usually is directly proportional to the supply voltage. For instance, if we reduce the supply voltage from 9 Volts to 6 Volts the motor will run at half or lower the speed. The advantages used DC motor is provide excellent speed control for acceleration and deceleration with effective and simple torque control. The fact that the power supply of a DC motor connects directly to the field of the motor allows for precise voltage control, which is necessary with speed and torque control applications. In this project, the method use as controller is $741 \mathrm{Op}-\mathrm{Amp}$, Transistor for the electric current control to drive a motor. The expectation of this project is to get the precise the demanded speed and to drive a motor at that speed. A simple Dc motor use electricity and magnetic field for producing torque which rotate the motor. PMDC permanent magnet DC motor outperforms to AC motor because it provide better speed control on high torque loads and use in wide industrial application. Dc motors are more usable as it designed to use with batteries and solar cells energy sources, which provide portability where we required it and thus provide cost effective solution because it is not possible to have AC power supply in every place [2], DC motor show its response at both voltage and current. The applied voltage describes the speed of motor while current in the armature windings shows the torque. If applied load increased in the shaft of motor then in order to sustain its speed motor draw more current from supply and if supply is not able to provide enough current then motor speed will be affected. Generally it can be said that applied voltage effect speed while torque is controlled by current. Dc motor provides more effective results if chopping circuit is used. Low power DC motors usually use in lifting and transportation purposes as low power AC motors don ' t have good torque capability .DC motor used in railway engines, electric cars, elevators, robotic applications, car windows and wide verity of small appliances and complex industrial mixing process where torque cannot be compromised. There are several types of DC motor but most common are brushed DC motor, brushless DC motor, stepper motor and servo motor. These motors are discussed latter also DC motors have three winding techniques such as shunt DC motor, series DC motor, and compound DC motor. A DC motor is an electric motor that runs on direct current (DC) electricity. DC motors were used to run machinery, often eliminating the need for a local steam engine or internal combustion engine. DC motors can operate directly from rechargeable batteries, providing the motive power for the first electric vehicles. Today DC motors are still found in applications as small as toys and disk drives, or in large sizes to operate steel rolling mills and paper machines. Modern DC motors are nearly always operated in conjunction with power electronic devices A motor controller is a device or group of devices that serves to govern in some predetermined manner the performance of an electric motor. A motor controller might include a manual or automatic means for starting and stopping the motor, selecting forward or reverse rotation, selecting and regulating the speed, regulating or limiting the torque, and protecting against 
overloads and faults. Motor controllers can be manually, remotely or automatically operated. They may include only the means for starting and stopping the motor or they may include other functions. An electric motor controller can be classified by the type of motor it is to drive such as permanent magnet, servo, series, separately excited, and alternating current. A motor controller is connected to a power source such as a battery pack or power supply, and control circuitry in the form of analog or digital input signals. A small motor can be started by simply plugging it into an electrical receptacle or by using a switch or circuit breaker. A larger motor requires a specialized switching unit called a motor starter or motor contactor. When energized, a direct on line (DOL) starter immediately connects the motor terminals directly to the power supply. Reduced-voltage, star-delta or soft starters connect the motor to the power supply through a voltage reduction device and increase the applied voltage gradually or in steps. In smaller sizes a motor starter is a manually operated switch; larger motors, or those requiring remote or automatic control, use magnetic contactors. Very large motors running on medium voltage power supplies (thousands of volts) may use power circuit breakers as switching elements. A direct on line (DOL) or across the line starter applies the full line voltage to the motor terminals, the starters or cubicle locations can usually be found on an ELO drawing. This is the simplest type of motor starter. A DOL motor starter also contain protection devices, and in some cases, condition monitoring. Smaller sizes of direct on-line starters are manually operated; larger sizes use an electromechanical contactor (relay) to switch the motor circuit. Solid-state direct on line starters also exist. A direct on line starter can be used if the high inrush current of the motor does not cause excessive voltage drop in the supply circuit. The maximum size of a motor allowed on a direct on line starter may be limited by the supply utility for this reason. For example, a utility may require rural customers to use reduced-voltage starters for motors larger than $10 \mathrm{~kW}$. DOL starting is sometimes used to start small water pumps, compressors, fans and conveyor belts. In the case of an asynchronous motor, such as the 3phase squirrel-cage motor, the motor will draw a high starting current until it has run up to full speed. This starting current is typically 6-7 times greater than the full load current. To reduce the inrush current, larger motors will have reduced-voltage starters or variable speed drives in order to minimize voltage dips to the power supply. A reversing starter can connect the motor for rotation in either direction. Such a starter contains two DOL circuits-one for clockwise operation and the other for counter-clockwise operation, with mechanical and electrical interlocks to prevent simultaneous closure. For three phase motors, this is achieved by transposing any two phases. Single phase AC motors and direct-current motors require additional devices for reversing rotation. Two or more contactors may be used to provide reduced voltage starting of a motor. By using an autotransformer or a series inductance, a lower voltage is present at the motor terminals [4], reducing starting torque and inrush current. Once the motor has come up to some fraction of its fullload speed, the starter switches to full voltage at the motor terminals. Since the autotransformer or series reactor only carries the heavy motor starting current for a few seconds, the devices can be much smaller compared to continuously rated equipment. The transition between reduced and full voltage may be based on elapsed time, or triggered when a current sensor shows the motor current has begun to reduce. An autotransformer starter was patented in 1908. An adjustable-speed drive (ASD) or variable-speed drive (VSD) is an interconnected combination of equipment that provides a means of driving and adjusting the operating speed of a mechanical load. An electrical adjustable-speed drive consists of an electric motor and a speed controller or power converter plus auxiliary devices and equipment. In common usage, the term "drive" is often applied to just the controller. An Intelligent Motor Controls (IMC) uses a microprocessor to control power electronic devices used for motor control. IMCs monitor the load on a motor and accordingly match motor torque to motor load. This is accomplished by reducing the voltage to the AC terminals and at the same time lowering current and kVAR. This can provide a measure of energy efficiency improvement for motors that run under light load for a large part of the time, resulting in less heat, noise, and vibrations generated by the motor. Starters using magnetic contactors usually derive the power supply for the contactor coil from the same source as the motor supply. An auxiliary contact from the contactor is used to maintain the contactor coil energized after the start command for the motor has been released. If a momentary loss of supply voltage occurs, the contactor will open and not close again until a new start command is given. This prevents restarting of the motor after a power failure. This connection also provides a small degree of protection against low power supply voltage and loss of a phase. However since contactor coils will hold the circuit closed with as little as $80 \%$ of normal voltage applied to the coil, this is not a primary means of protecting motors from low voltage operation. A motor control center (MCC) is an assembly of one or more enclosed sections having a common power bus and principally containing motor control units. Motor control centers are in modern practice a factory assembly of several motor starters. A motor control center can include variable frequency drives, programmable controllers, and metering and may also be the electrical service entrance for the building. Motor control centers are usually used for low voltage three-phase alternating current motors from $208 \mathrm{~V}$ to $600 \mathrm{~V}$. Mediumvoltage motor control centers are made for large motors running at $2300 \mathrm{~V}$ to around $15000 \mathrm{~V}$, using vacuum contactors for switching and with separate compartments for power switching and control. Motor control centers have been used since 1950 by the automobile 
manufacturing industry which used large numbers of electric motors. Today they are used in many industrial and commercial applications. Where very dusty or corrosive processes are used, the motor control center may be installed in a separate air-conditioned room, but often an MCC will be on the factory floor adjacent to the machinery controlled. A motor control center consists of one or more vertical metal cabinet sections with power bus and provision for plug-in mounting of individual motor controllers. Very large controllers may be bolted in place but smaller controllers can be unplugged from the cabinet for testing or maintenance. Each motor controller contains a contactor or a solid-state motor controller; overload relays to protect the motor, fuses or a circuit breaker to provide short-circuit protection, and a disconnecting switch to isolate the motor circuit. Three-phase power enters each controller through separable connectors. The motor is wired to terminals in the controller. Motor control centers provide wire ways for field control and power cables. Each motor controller in an MCC can be specified with a range of options such as separate control transformers, pilot lamps, control switches, extra control terminal blocks, various types of thermal or solid-state overload protection relays, or various classes of power fuses or types of circuit breakers. A motor control center can either be supplied ready for the customer to connect all field wiring, or can be an engineered assembly with internal control and interlocking wiring to a central control terminal panel board or programmable controller. Motor control centers (MCC) usually sit on floors, which are often required to have a fire-resistance rating. Fire stops may be required for cables that penetrate fire-rated floors and walls. The purpose to control the speed of motor is because there are many application in which user needs to change the speed of motor to get certain tasks. In robotics speed control is very important because only robots can work properly if motors speed is controlled in precise way. One example is CNC machine in which $1 \mathrm{~mm}$ of error cannot be compromised so dc motor in such case provides exact speed control. The speed control normally done by Feedback speed controllers or closed loop speed controllers. So it is oblivious that without speed controllers we cannot get our goal in robotic and industrial application. Generally, the rotational speed of a DC motor is proportional to the EMF in its coil (= the voltage applied to it minus voltage lost on its resistance), and the torque is proportional to the current. Speed control can be achieved by variable battery tapping, variable supply voltage, resistors or electronic controls. The direction of a wound field DC motor can be changed by reversing either the field or armature connections but not both. This is commonly done with a special set of contactors (direction contactors). The effective voltage can be varied by inserting a series resistor or by an electronically controlled switching device made of thyristors, transistors, or formerly, mercury arc rectifiers, in a circuit known as a chopper, the average voltage applied to the motor is varied by switching the supply voltage very rapidly. As the "on" to "off" ratio is varied to alter the average applied voltage, the speed of the motor varies. The percentage "on" time multiplied by the supply voltage gives the average voltage applied to the motor. Therefore, with a $100 \mathrm{~V}$ supply and a $25 \%$ "on" time, the average voltage at the motor will be $25 \mathrm{~V}$. During the "off" time, the armature's inductance causes the current to continue through a diode called a "fly back diode", in parallel with the motor. At this point in the cycle, the supply current will be zero, and therefore the average motor current will always be higher than the supply current unless the percentage "on" time is $100 \%$. At $100 \%$ "on" time, the supply and motor current are equal. The rapid switching wastes less energy than series resistors. This method is also called pulse-width modulation (PWM) and is often controlled by a microprocessor. An output filter is sometimes installed to smooth the average voltage applied to the motor and reduce motor noise. Since the serieswound DC motor develops its highest torque at low speed, it is often used interaction applications such as electric locomotives, and trams. Another application is starter motors for petrol and small diesel engines. Series motors must never be used in applications where the drive can fail (such as belt drives). As the motor accelerates, the armature (and hence field) current reduces. The reduction in field causes the motor to speed up until it destroys itself [6]. This can also be a problem with railway motors in the event of a loss of adhesion since, unless quickly brought under control, the motors can reach speeds far higher than they would do under normal circumstances. This can not only cause problems for the motors themselves and the gears, but due to the differential speed between the rails and the wheels it can also cause serious damage to the rails and wheel treads as they heat and cool rapidly. Field weakening is used in some electronic controls to increase the top speed of an electric vehicle. The simplest form uses a contactor and field-weakening resistor; the electronic control monitors the motor current and switches the field weakening resistor into circuit when the motor current reduces below a preset value (this will be when the motor is at its full design speed). Once the resistor is in circuit, the motor will increase speed above its normal speed at its rated voltage. When motor current increases, the control will disconnect the resistor and low speed torque is made available. One interesting method of speed control of a DC motor is the Ward Leonard control. It is a method of controlling a DC motor (usually a shunt or compound wound) and was developed as a method of providing a speed-controlled motor from an AC supply, though it is not without its advantages in DC schemes. The AC supply is used to drive an AC motor, usually an induction motor that drives a DC generator or dynamo. The DC output from the armature is directly connected to the armature of the DC motor (sometimes but not always of identical construction). The shunt field windings of both DC machines are 
independently excited through variable resistors. Extremely good speed control from standstill to full speed, and consistent torque, can be obtained by varying the generator and/or motor field current. This method of control was the de facto method from its development until it was superseded by solid state thyristor systems. It found service in almost any environment where good speed control was required, from passenger lifts through to large mine pit head winding gear and even industrial process machinery and electric cranes. Its principal disadvantage was that three machines were required to implement a scheme (five in very large installations, as the DC machines were often duplicated and controlled by a tandem variable resistor). In many applications, the motor-generator set was often left permanently running, to avoid the delays that would otherwise be caused by starting it up as required. Although electronic (thyristor) controllers have replaced most small to medium Ward-Leonard systems, some very large ones (thousands of horsepower) remain in service. The field currents are much lower than the armature currents, allowing a moderate sized thyristor unit to control a much larger motor than it could control directly. For example, in one installation, a $300 \mathrm{amp}$ thyristor unit controls the field of the generator. The generator output current is in excess of 15,000 amperes, which would be prohibitively expensive (and inefficient) to control directly with thyristors. To extend a D.C. motor's service life, protective devices and motor controllers are used to protect it from mechanical damage, excessive moisture, high dielectric stress and high temperature or thermal overloading. These protective devices sense motor fault conditions and either annunciate an alarm to notify the operator or automatically de-energize the motor when a faulty condition occurs. For overloaded conditions, motors are protected with thermal overload relays. Bi-metal thermal overload protectors are embedded in the motor's windings and made from two dissimilar metals. They are designed such that the bimetallic strips will bend in opposite directions when a temperature set point is reached to open the control circuit and de-energize the motor. Heaters are external thermal overload protectors connected in series with the motor's windings and mounted in the motor contactor. Solder pot heaters melt in an overload condition, which cause the motor control circuit to de-energize the motor. Bimetallic heaters function the same way as embedded bimetallic protectors. Fuses and circuit breakers are over current or short circuit protectors. Ground fault relays also provide over current protection. They monitor the electrical current between the motor's windings and earth system ground. In motor-generators, reverse current relays prevent the battery from discharging and motorizing the generator [7]. Since D.C. motor field loss can cause a hazardous runaway or over speed condition, loss of field relays are connected in parallel with the motor's field to sense field current. When the field current decreases below a set point, the relay will deenergize the motor's armature. A locked rotor condition prevents a motor from accelerating after its starting sequence has been initiated. Distance relays protect motors from locked-rotor faults. Under voltage motor protection is typically incorporated into motor controllers or starters. In addition, motors can be protected from over voltages or surges with isolation transformers, power conditioning equipment, MOVs, arrestors and harmonic filters. Environmental conditions, such as dust, explosive vapors, water, and high ambient temperatures, can adversely affect the operation of a DC motor. To protect a motor from these environmental conditions, the National Electrical Manufacturers Association (NEMA) and the International Electro technical Commission (IEC) have standardized motor enclosure. Designs based upon the environmental protection they provide from contaminants. Every electric motor has to have some sort of driver. The motor driver will have differing features and complexity depending on the task that the motor will be performing. The simplest case is a switch to connect a motor to a power source, such as in small appliances or power tools. The switch is manually operated or may be a relay or contactor connected to some form of drive to manually start and stop the motor. The potentiometer may have several positions to select different connections of 741 Op-amp. This allows reduced-voltage starting of the motor, reversing control or selection of multiple speeds. Overload and over current protection may be omitted in very small motor controllers, which relay on the supplying circuit to have over current protection. Small motors may have built-in overload devices to manually open the circuit on overload. Larger motors have a protective overload relay. A motor driver may also include limit switches or other devices to protect the driven machinery. The speed of the motor has to be increased from zero and brought to the operating speed. The operating speed itself should be varied as per the requirements of the load. This is called speed control. The running motor has to be brought to rest, by decelerating the same. This is called braking. The torque speed characteristics of the machine are modified to achieve these as it is assumed that the variation in the characteristics of the load is either not feasible or desirable. Hence the methods that are available for modifying the torque speed characteristics and the actual variations in the performance that these methods bring about are of great importance. When more than one method is available for achieving the same objective then other criteria like, initial cost, running cost, efficiency and ease operation are also applied for the evaluation of the methods. Due to the absence of equipment like transformer, dc motor operation in 99 general is assumed to be off a constant voltage dc. supply. For the motor to start, the torque developed by the motor at zero speed must exceed that demanded by the load. Then TM - TL will be positive so also is $\mathrm{d}$ ! /dt and the motor accelerate. The induced emf at starting point is zero as the $!=0$ the armature current with rated applied voltage is given by $\mathrm{V} / \mathrm{Ra}$ where $\mathrm{Ra}$ is armature circuit resistance. 
Normally the armature resistance of a dc motor is such as to cause 1 to 5 percent drop at full load current. Hence the starting current tends to rise to several times the full load current. The same can be told of the torque if full flux is already established. The dc motor instantly picks up the speed. As the speed increases the induced emf appears across the terminals opposing the applied voltage. The current drawn from the mains thus decreases, so also the torque. This continues till the load torque and the motor torque are equal to each other. Motor tends to run continuously at this speed as the acceleration is zero at this point of operation. The starting is now discussed with respect to specific motors. When a motor is switched off it 'coasts' to rest under the action of frictional forces. Braking is employed when rapid stopping is required. In many cases mechanical braking is adopted. The electric braking may be done for various reasons such as those mentioned below: In many cases electric braking makes more brake power available to the braking process where mechanical brakes are applied. This reduces the wear and tear of the mechanical brakes and reduces the frequency of the replacement of these parts. By recovering the mechanical energy stored in the rotating parts and pumping it into the supply lines the overall energy efficiency is improved. This is called regeneration. Where the safety of the personnel or the equipment is at stake the machine may be required to stop instantly. Extremely large brake power is needed under those conditions. Electric braking can help in these situations also. In processes where frequent starting and stopping is involved the process time requirement can be reduced if braking time is reduced. The reduction of the process time improves the throughput. Basically the electric braking involved is fairly simple. The electric motor can be made to work as a generator by suitable terminal conditions and absorb mechanical energy. This converted mechanical power is dissipated/used on the electrical network suitably.

\section{PCB Design of DC Driver Circuit}

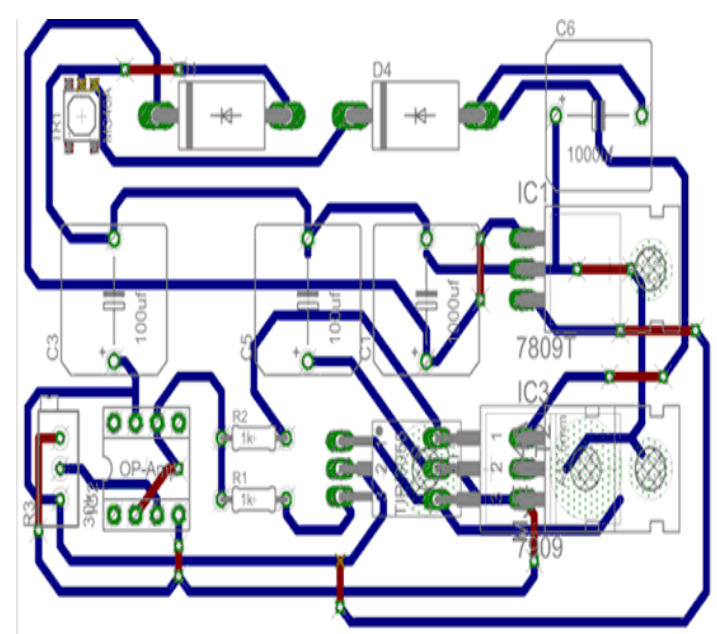

Fig. 3.3.1. PCB Design of dc driver circuit.

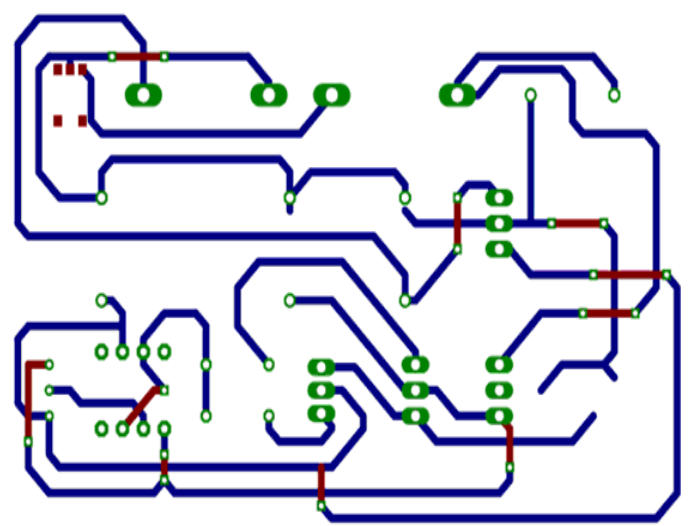

Fig. 3.3.2. $P C B$ Design of dc driver circuit.

As we all know any invention of latest technology cannot be activated without the source of power. So in this fast moving world, we deliberately need a proper power source which will be apt for a particular requirement. All the electronic components starting from diode to LM IC's only work with a DC supply ranging from $+9 v$ and $-9 v$. The voltage supply gives from the $220 \mathrm{ac}$ voltage. First by using by step down transformer the $220 \mathrm{v}$ ac is converted into the $12 \mathrm{v}$ ac. This $12 \mathrm{v}$ ac current is converted into $12 \mathrm{dc}$ voltage by rectifying with diode. We use two voltage regulators IC- LM 7809 and IC-LM 7909. One is IC-L7809 which stables the voltage at +9 volt and other one is IC-LM 7909 which stables the voltage at -9 volt. In the power supply unit, rectification is normally achieved using a solid state diode. Diode has the property that will let the electron flow easily in one direction at proper biasing condition. As $\mathrm{AC}$ is applied to the diode, electrons only flow when the anode and cathode is negative. Reversing the polarity of voltage will not permit electron flow. A commonly used circuit for supplying large amounts of DC power is the bridge Rectifier. A bridge rectifier of four diodes (4*IN4007) are used to achieve full wave rectification. Two diodes will conduct during the negative cycle and the other two will conduct during the positive half cycle. The DC voltage appearing across the output terminals of the bridge rectifier will be less than $90 \%$ of the applied rms value. Normally one alteration of the input voltage will reverse the polarities. Opposite ends of the transformer will therefore always be $180 \mathrm{deg}$ out of phase with each other. For a positive cycle, two diodes are connected to the positive voltage at the top winding and only one diode conducts. At the same time one of the other two diodes conducts for the negative voltage that is applied from the bottom winding due to the forward bias for that diode. In this circuit due to positive half cycle D1 \& D2 will conduct to give $10.8 \mathrm{v}$ pulsating DC. The DC output has a ripple frequency of $100 \mathrm{~Hz}$. Since each alteration produces a resulting output pulse frequency $=2 * 50 \mathrm{~Hz}$. The output obtained is not a pure DC and therefore filtration has to be done. Filter circuits, which is usually a capacitor acting as a surge arrester, always follow the rectifier unit. This capacitor is also called as a decoupling capacitor or a 
bypassing capacitor, which is used not only to 'short' the ripple with frequency of $120 \mathrm{~Hz}$ to ground but also to leave the frequency of the DC to appear at the output. A load resistor R1 is connected so that a reference to the ground is maintained. $\mathrm{C} 1 \mathrm{R} 1$ is for bypassing ripples and $\mathrm{C} 2 \mathrm{R} 2$ is used as a low pass filter, i.e. it passes only low frequency signals and bypasses high frequency signals. The load resistor should be $1 \%$ to $2.5 \%$ of the load. $1000 \mathrm{uf} / 25 \mathrm{v}$ : for the reduction of ripples from the pulsating. $100 \mathrm{uf} / 25 \mathrm{v}$ : for maintaining the stability of the voltage at the load side 0.1uf: for bypassing the high frequency disturbances. The voltage regulators play an important role in any power supply unit. The primary purpose of a regulator is to aid the rectifier and filter circuit in providing a constant DC voltage to the device. Power supplies without regulators have an inherent problem of changing DC voltage values due to variations in the load or due to fluctuations in the AC liner voltage. With a regulator connected to the DC output, the voltage can be maintained within a close tolerant region of the desired output. IC7809 and 7909 is used in this project for providing $+9 \mathrm{v}$ and $-9 \mathrm{v}$ DC supply. An operational amplifier ("op-amp") is a Dc -coupled highgain electronic voltage amplifier with a differential input and, usually, a single-ended output. An op-amp produces an output voltage that is typically hundreds of thousands of times larger than the voltage difference between its input terminals. Operational amplifiers had their origins in analog computers where they were used to do mathematical operations in many linear, non-linear and frequency-dependent circuits. Characteristics of a circuit using an op-amp are set by external components with little dependence on temperature changes or manufacturing variations in the op-amp itself, which makes op-amps popular building blocks for circuit design. Op-amps are among the most widely used electronic devices today, being used in a vast array of consumer, industrial, and scientific devices. Many standard IC op-amps cost only a few cents in moderate production volume; however some integrated or hybrid operational amplifiers with special performance specifications may cost over \$100 US in small quantities. Op-amps may be packaged as components, or used as elements of more complex integrated circuits. The op-amp is one type of differential amplifier. Other types of differential amplifier include the fully differential amplifier (similar to the op-amp, but with two outputs), the instrumentation amplifier (usually built from three opamps), the isolation amplifier (similar to the instrumentation amplifier, but with tolerance to commonmode voltages that would destroy an ordinary op-amp), and negative feedback amplifier (usually built from one or more op-amps and a resistive feedback network).The power supply pins $\left(V_{\mathrm{S}+}\right.$ and $\left.V_{\mathrm{S}-}\right)$ can be labeled in different ways (See IC power supply pins). Despite different labeling, the function remains the same - to provide additional power for amplification of the signal. Often these pins are left out of the diagram for clarity, and the power configuration is described or assumed from the circuit. A component level diagram of the common 741 opamp. Dotted lines outline: current mirrors (red); differential amplifier (blue); class a gain stage (magenta); voltage level shifter (green); output stage (cyan). Though designs vary between products and manufacturers, all op-amps have basically the same internal structure, which consists of three stages: Differential amplifier — provides low noise amplification, high input impedance, usually a differential output. Voltage amplifier - provides high voltage gain, a single-pole frequency roll-off, usually single-ended output. Output amplifier — provides high current driving capability, low output impedance, current limiting and short circuit protection circuitry. IC op-amps as implemented in practice are moderately complex integrated circuits. A typical example is the ubiquitous 741 op-amp designed by Dave Fullagar in Fairchild Semiconductor after the remarkable Widlar LM301. Thus the basic architecture of the 741 is identical to that of the 301 . The input stage is a composed differential amplifier with a complex biasing circuit and a current mirror active load. It is implemented by two cascaded stages satisfying the conflicting requirements. The first stage consists of the NPN-based input emitter followers Q1 and Q2 that provide high input impedance. The next is the PNP-based common base pair Q3 and Q4 that eliminates the undesired Miller effect, shifts the voltage level downwards and provides a sufficient voltage gain to drive the next class A amplifier. The PNP transistors also help to increase the reverse $V_{\mathrm{BE}}$ rating (the base-emitter junctions of the NPN transistors Q1 and Q2 break down at around $7 \mathrm{~V}$ but the PNP transistors Q3 and Q4 have breakdown voltages around $50 \mathrm{~V}$ ). The classical emitter-coupled differential stage is biased from the side of the emitters by connecting a constant current source to them. The series negative feedback (the emitter degeneration) makes the transistors act as voltage stabilizers; it forces them to adjust their $V_{\mathrm{BE}}$ voltages so that to pass the current through their collector-emitter junctions. As a result, the quiescent current is $\beta$-independent. Here, the Q3/Q4 emitters are already used as inputs. Their collectors are separated and cannot be used as inputs for the quiescent current source since they behave as current sources. So, the quiescent current can be set only from the side of the bases by connecting a constant current source to them. To make it not depend on $\beta$ as above, a negative but parallel feedback is used. For this purpose, the total quiescent current is mirrored by Q8-Q9 current mirror and the negative feedback is taken from the Q9 collector. Now it makes the transistors Q1-Q4 adjust their $V_{\mathrm{BE}}$ voltages so that to pass the desired quiescent current. The effect is the same as at the classical emitter-coupled pair - the quiescent current is $\beta$-independent. It is interesting fact that "to the extent that all PNP $\beta$ s match, this clever circuit generates just the right $\beta$-dependent base current to produce a $\beta$-independent collector current". The biasing base currents are usually provided only by the negative power 
supply; they should come from the ground and enter the bases [9]. But to ensure maximum high input impedances, the biasing loops are not internally closed between the base and ground; it is expected they will be closed externally by the input sources. So, the sources have to be galvanic (DC) to ensure paths for the biasing currents and low resistive enough (tens or hundreds kilo ohms) to not create significant voltage drops across them. Otherwise, additional DC elements should be connected between the bases and the ground (or the positive power supply). The quiescent current is set by the $39 \mathrm{k} \Omega$ resistor that is common for the two current mirrors Q12-Q13 and Q10$\mathrm{Q} 11$. The current determined by this resistor acts also as a reference for the other bias currents used in the chip. The Widlar current mirror built by Q10, Q11, and the $5 \mathrm{k} \Omega$ resistor produces a very small fraction of $\mathrm{I}_{\text {ref }}$ at the Q10 collector. This small constant current through Q10's collector supplies the base currents for Q3 and Q4 as well as the Q9 collector current. The Q8/Q9 current mirror tries to make Q9 collector current the same as the Q3 and Q4 collector currents and succeeds with the help of the negative feedback. The Q9 collector voltage changes until the ratio between the Q3/Q4 base and collector currents becomes equal to $\beta$. Thus Q3 and Q4's combined base currents (which are of the same order as the overall chip's input currents) are a small fraction of the already small Q10 current. Thus the quiescent current is set by Q10-Q11 current mirror without using a current-sensing negative feedback. The voltage-sensing negative feedback only helps this process by stabilizing Q9 collector (Q3/Q4 base) voltage. The feedback loop also isolates the rest of the circuit from common-mode signals by making the base voltage of Q3/Q4 follow tightly $2 V_{\mathrm{BE}}$ below the higher of the two input voltages. The differential amplifier formed by Q1-Q4 drives an active load implemented as an improved current mirror (Q5-Q7) whose role is to convert the differential current input signal to a single ended voltage signal without the intrinsic 50\% losses and to greatly increase the gain. This is achieved by copying the input signal from the left to the right side where the magnitudes of the two input signals add (Widlar used the same trick in $\mu \mathrm{A} 702$ and $\mu \mathrm{A} 709$ ). For this purpose, the input of the current mirror (Q5 collector) is connected to the left output (Q3 collector) and the output of the current mirror (Q6 collector) is connected to the right output of the differential amplifier (Q4 collector). Q7 increases the accuracy of the current mirror by decreasing the amount of signal current required from Q3 to drive the bases of Q5 and Q6. The input voltage sources are connected through two "diode" strings, each of them consisting of two connected in series base-emitter junctions (Q1-Q3 and Q2-Q4), to the common point of $\mathrm{Q} 3 / \mathrm{Q} 4$ bases. So, if the input voltages change slightly in opposite directions, Q3/Q4 bases stay at relatively constant voltage and the common base current does not change as well; it only vigorously steers between Q3/Q4 bases and makes the common quiescent current distribute between Q3/Q4 collectors in the same proportion. The current mirror inverts Q3 collector current and tries to pass it through Q4. In the middle point between Q4 and Q6, the signal currents (current changes) of Q3 and Q4 are subtracted. In this case (differential input signal), they are equal and opposite. Thus, the difference is twice the individual signal currents $(\Delta I-(-\Delta I)=2 \Delta I)$ and the differential to single ended conversion is completed without gain losses. The open circuit signal voltage appearing at this point is given by the product of the subtracted signal currents and the total circuit impedance (the paralleled collector resistances of Q4 and Q6). Since the collectors of Q4 and Q6 appear as high differential resistances to the signal current $(\mathrm{Q} 4$ and Q6 behave as current sources), the open circuit voltage gain of this stage is very high. More intuitively, the transistor Q6 can be considered as a duplicate of Q3 and the combination of Q4 and Q6 can be thought as of a varying voltage divider composed of two voltage-controlled resistors. For differential input signals, they vigorously change their instant resistances in opposite directions but the total resistance stays constant (like a potentiometer with quickly moving slider). As a result, the current stays constant as well but the voltage at the middle point changes vigorously. As the two resistance changes are equal and opposite, the effective voltage change is twice the individual change. The base current at the inputs is not zero and the effective differential input impedance of a 741 is about $2 \mathrm{M} \Omega$. The "offset null" pins may be used to place external resistors in parallel with the two $1 \mathrm{k} \Omega$ resistors (typically in the form of the two ends of a potentiometer) to adjust the balancing of the Q5/Q6 current mirror and thus indirectly control the output of the op-amp when zero signal is applied between the inputs. Common mode If the input voltages change in the same direction, the negative feedback makes Q3/Q4 base voltage follow (with $2 V_{\mathrm{BE}}$ below) the input voltage variations. Now the output part (Q10) of Q10-Q11 current mirror keeps up the common current through Q9/Q8 constant in spite of varying voltage. Q3/Q4 collector currents and accordingly, the output voltage in the middle point between Q4 and Q6, remain unchanged. The section outlined in magenta is the class a gain stage. The top-right current mirror Q12/Q13 supplies this stage by a constant current load, via the collector of Q13 that is largely independent of the output voltage. The stage consists of the two NPN transistors Q15/Q19 connected in a Darlington configuration and uses the output side of a current mirror as its collector (dynamic) load to achieve high gain. The transistor Q22 prevents this stage from saturating by diverting the excessive Q15 base current (it acts as a Baker clamp). The $30 \mathrm{pF}$ capacitor provides frequency selective negative feedback around the class a gain stage as a means of frequency compensation to stabilize the amplifier in feedback configurations. This technique is called Miller compensation and functions in a similar manner to an opamp integrator circuit. It is also known as 
'dominant pole compensation' because it introduces a dominant pole (one which masks the effects of other poles) into the open loop frequency response. This pole can be as low as $10 \mathrm{~Hz}$ in a 741 amplifier and it introduces a $-3 \mathrm{~dB}$ loss into the open loop response at this frequency. This internal compensation is provided to achieve unconditional stability of the amplifier in negative feedback configurations where the feedback network is non-reactive and the closed loop gain is unity or higher. Hence, the use of the operational amplifier is simplified because no external compensation is required for unity gain stability; amplifiers without this internal compensation such as the 748 may require external compensation or closed-loop gains significantly higher than unity. DC Motors have speed control capabilities, which means that speed, torque and even direction of rotation can be changed at anytime to meet new condition. This system will be able to control the DC Motor speed at desired speed regardless of the changes of load. DC Motor in inherently bidirectional. Hence there is no problem to forward and reverse the direction. The speed of DC Motor has been successfully controlled by using Op-Amp 741 controlled technique. DC Driver is less complex with a single power conversion from AC to DC. For servo application, DC drives is still popular because of good dynamic response and ease of control. The fabrication results under varying reference speed and varying load are also studied and analyzed. The model shows good results under all conditions employed during fabrication.

\section{Conclusion}

If this DC Motor Drive system can be made commercially, the cost will comparatively be reduced. The results from the fabrication were never likely to occur in real-life condition due to the response times and condition of the actual motor [8]. In near future this system can control the motor speed by using Op-Amp 741. For low power applications the cost of DC motor drives is still economic.

\section{References}

[1] B.L Theraja \& A.K Theraja, AC \& DC Machines, Khanna Publishers, New Delhi, 2003.

[2] Bimbhra, P.S., Power Electronics, Khanna Publishers, New Delhi, 2006.

[3] Dubey, G.K., Fundamentals of Electrical Drives. New Delhi, Narosa Publishing House, 2000.

[4] Rashid, M.H., Power Electronics, Prentice Hall of India, New Delhi, 1993.

[5] Infineon Technologies, Basic DC motor speed PID control with the Infineon Technologies, 1996.

[6] Salam Dr. Zainal, UTJMB, Power Electronics and Drives (Version 3-2003).

[7] Gopal, M., Control Systems, Principles and Design. New Delhi, Tata McGraw Hill Publishing Company limited 2008.

[8] J.D Nicould, O Matthey and G.Caprari, "Control and Analysis of Low Inertia Miniature Synchronous Motors". International Symposium on Micromehantronics and Human Science, 1998.

[9] Advanced PM brushless DC motor control and system for electric vehicle, Fang Lin Luo; Hock Guan Yeo, 1990.

[10] Delmar, Herman, Stephen L. Cengage Learning, Electric Motor Control, 9th ed. 2009.

[11] http://www.electronics-project-design.com. 